\title{
Arthropathy and cutaneous manifestations in a 28-year-old patient with cystic fibrosis
}

\author{
S. Lampert1, S. Pour Schahin1, G.H. Wiest1, E.G. Hahn1, J.H. Ficker2
}

\begin{abstract}
Arthropathy and Cutaneous Manifestations in a 28-Year-old Patient with Cystic Fibrosis. S. Lampert, S. Pour Schahin, G.H. Wiest, E.G. Hahn, J.H. Ficker.

We report on a 28-year-old man with known cystic fibrosis who presented with pain and cutaneous nodules in the elbow joints. His symptoms had appeared episodically in the previous months, they were always self-limiting, and independent of pulmonary exacerbations. A radiograph of the joints was unremarkable. These findings fit well with a special form of CF-related arthritis. As in the case of classical CF arthritis, the treatment to be considered is, in particular, symptomatic administration of non-steroidal anti-inflammatory drugs and possibly glucocorticoids. Al-
\end{abstract}

so under discussion as a further possibility is the use of antibiotics. Our patient has always refused medication. The condition again proved to be self-limiting.

In contrast to the classical form of $\mathrm{CF}$ arthritis, the special form is not associated with either joint swelling or local warmth. In the presence of arthritic symptoms in CF patients, consideration must always be given to a hypertrophic pulmonary osteoarthropathy. The latter, however, shows typical radiological changes and is exacerbated by lung infections.

The presence of arthritic pathologies in a patient with CF further underscores the fact that $\mathrm{CF}$ is a multiorgan morbid condition.

Monaldi Arch Chest Dis 2006; 65: 2, 114-115.

Keywords: Cystic fibrosis, arthritis, arthropathy, cutaneous manifestations.

1 Medical Department 1, University of Erlangen-Nuremberg; Erlangen,

2 Medical Department 3, Klinikum Nürnberg; Nuremberg, Germany.

Correspondence: Dr. med. Sabine Lampert; Medical Department 1; University of Erlangen-Nuremberg; Ulmenweg 18; D- 91054 Erlangen; Germany; e-mail: Sabine.Lampert@medl.imed.uni-erlangen.de

In patients suffering from cystic fibrosis $(\mathrm{CF})$, two different articular pathologies have been described: so-called CF arthritis and hypertrophic pulmonary osteoarthropathy [1]. The incidence of each of these entities in cystic fibrosis patients is around $10 \%[1,2]$. We report herein on a 28 -yearold man with CF who developed a special form of CF-related arthritis.

\section{Case report}

A 28-year-old man with cystic fibrosis $(\Delta$ F508 homozygote) complained of painful elbow joint symptoms associated with episodic limitation of movement during the previous three months. Neither joint swelling nor erythema was seen, but he developed clearly palpable cutaneous nodules over the joints affected by the attacks. It was not possible to establish any temporal relationship between the self-limiting symptomatology persisting for between 1 and 3 weeks, and pulmonary infections.

In addition to a $\mathrm{CF}$-associated distal intestinal obstruction syndrome (DIOS) two years earlier, the patient has a 10 -year history of schizophrenia. The patient's regular medication comprised pancreas enzymes, bronchodilators and neuroleptic agents. The patient smoked (10 py), and his atten- dance at the $\mathrm{CF}$ clinic for routine surveillance examinations was irregular. Physical examination revealed an underweight patient $(166 \mathrm{~cm}, 44 \mathrm{~kg})$ in a reduced state of health. In addition to clubbed fingers and toes, crackles were also heard over both lungs. Cardiac status and abdominal status were both unremarkable. Laboratory investigations showed no elevation of inflammatory parameters or immunological alterations. The chest radiograph revealed typical signs that had remained unchanged since the preceding radiograph. This meant that, despite a diminishment in the parameter forced one second capacity $\left(\mathrm{FEV}_{1} 16 \%\right.$ pred.; VC $27 \%$ pred.), his state of health was recorded as poor but stable. As reported by the patient, firm, non-mobile, non-tender nodules were present, bilaterally, in the region of the dorsum of the proximal forearm (fig. 1). Neither erythema nor swelling of the elbow joint was to be seen. Although active mobility was not restricted, the patient experienced pain on passive movement of the respective joints.

At ultrasonography, the nodular changes, measuring up to $25 \times 6 \mathrm{~mm}$, presented as echo-poor structures in direct contact with the periosteum. From the radiograph obtained in the region of the cutaneous manifestations (elbow joints; proximal part of the forearm), no pathological intra- or peri- 


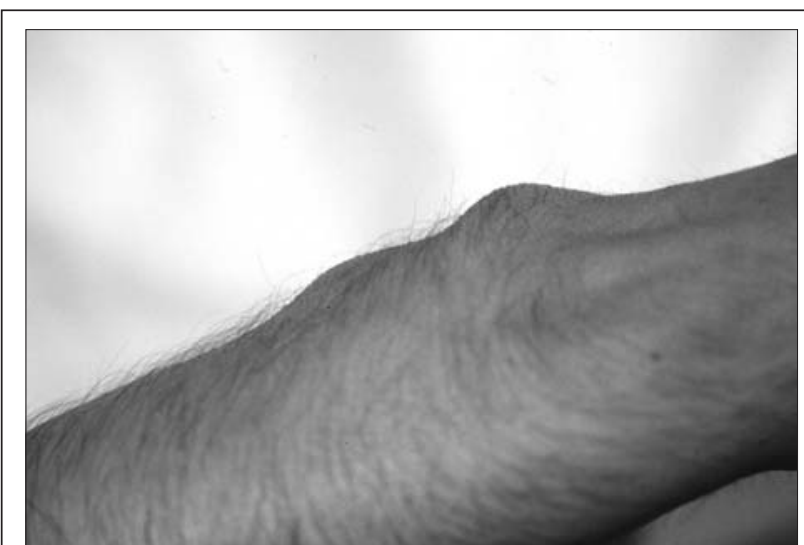

Fig. 1.

articular calcifications were seen. The bony structures were in correct anatomical alignment, articulation and mineralisation were normal, and the joint spaces regular; there were no signs of regenerative or inflammatory joint changes.

\section{Discussion}

The clinical picture encompassing nodular cutaneous manifestations and painful joints, and unremarkable radiographic findings, are readily compatible with a variant of CF-related arthritis [3]. The latter is differentiated from the classical form of CF-related arthritis in which swelling of the joints and local warming also occur [1] and should therefore probably be termed arthropathy rather than arthritis. The appearance of this type of arthritis during the course of cystic fibrosis is independent of the severity of the pulmonary manifestations [1]. The literature also reports a number of cases with considerably impaired primary function, as in our own patient [2]. A wide variety of cutaneous manifestations is found in up to $40 \%$ of patients with CF-related arthritides [2]. Such manifestations may be nodular changes, erythema or maculopapillary exanthems, which, as in the case of our own patient, are frequently to be found in the immediate neighbourhood of the affected joints [4].

Apart from these conspicuous nodules, our patient differs from most cases previously described as he was relatively "old", and showed no signs of inflammation. All other symptoms and the course of the disease were identical with those of the case histories of CF-related arthritis patients in the literature [1-11].

CF-related arthritis must be distinguished from hypertrophic pulmonary osteoarthropathy (HPOA). In the latter, also commonly associated with CF and joint pain, the articular and osseous symptoms typically increase appreciably in association with lung infections [1]. In contrast, the differential diagnosis of the two CF-related arthropathies is facilitated by the presence in HPOA of radiological changes, such as thickening of the periosteum.

To date, the pathogenesis of $\mathrm{CF}$ arthritis has not been unequivocally clarified. A possible pathogenetic mechanism is the deposition of immune complexes in the synovia [5]. Furthermore, a triggering role of colonisation of with Pseudomonas aeruginosa deep in the lower airways, for example, has been considered [12]. Unfortunately, since no data on such colonization in the patient has been provided in previous publications, a relevant comparison is not possible. Nor is the underlying cause of the nodular cutaneous manifestations clear. The prognosis of the disease is usually good.

Proposed treatment comprises the symptomatic administration of non-steroidal anti-inflammatory drugs and, where indicated, glucocorticoids [1]. Furthermore, there are also reports of successful treatment with intravenous antibiotics [6]. Admittedly, we currently have no prospective studies investigating the value of medication even when the untreated symptoms are of limited duration. In our own case, the response of the symptoms to drug treatment could not be investigated, since the patient always refused medication. During the further course of the disease, three more attacks of joint pain associated with nodular cutaneous manifestations occurred.

\section{References}

1. Johnson S, Knox AJ. Arthropathy in cystic fibrosis. Respir Med 1994; 88: 567-570.

2. Pertuiset E, Menkes CJ, Lenoir G, Jehanne M, Douchaine F, Guillot M. Cystic fibrosis arthritis. A report of five cases. Br J Rheumatol 1992; 31: 535-538.

3. Reinhardt D, Götz M, Kraemer R. Bewegungsapparat. In: Schoeni $\mathrm{MH}$, ed. Cystische Fibrose. Berlin: Springer-Verlag, 2001

4. Dixey J, Redington A, Butler RC, et al. The arthropathy of cystic fibrosis. Ann Rheum Dis 1988; 47: 218-223.

5. Lawrence JM, Moore TL, Madson KL, Rejent AJ, Osborn TG. Arthropathies of Cystic fibrosis: case reports and review of the literature. J Rheumatol Suppl 1993; 38:12-15.

6. Bowler IM, Littlewood JM. Episodic arthritis in cystic fibrosis. Lancet 1992; 340: 244.

7. Massie RJH, Towns SJ, Bernard E, Chaitow J, Howman-Giles R, Van Asperen PP. The musculo-skeletal complications of cystic fibrosis. I Paediatr Child Health 1998; 34: 467-470.

8. Rush PJ, Shore A, Coblentz C, Wilmot D, Corey M, Levinson H. The musculoskeletal manifestations of cystic fibrosis. Semin Arthritis Rheum 1986; 15: 213 225.

9. Bourke S, Rooney M, Fitzgerald M, Brenishan B. Episodic arthropathy in adult cystic fibrosis. $Q \mathrm{~J} \mathrm{Med}$ 1987; 64: 651-659.

10. Jones MM, Seilheimer DK, Pollack MS, Curry M, Crane MM, Rossen RD. Relationship of hypergammaglonulinemia, circulating immune complexes and histocompatibility antigen profiles in patients with cystic fibrosis. Am Rev Respir Dis 1989; 140: 1636-1639.

11. Turner MA, Baildam E, Patel L, David TF. Joint disorders in cystic fibrosis. $J$ R Soc $M$ 1997; 90:13-20.

12. Al-Shamma MR, McSharry C, McLeod K, McCruden EA, Stack BH. Role of heat shock proteins in the pathogenesis of cystic fibrosis arthritis. Thorax 1997; 52: 1056-1059. 